\title{
Índice de gradiente de canal: significados e diretrizes para aplicação
}

Adalto Gonçalves Lima*

\section{Resumo}

O índice de gradiente de canal é comumente utilizado para avaliação do controle exercido por fatores geológicos sobre o perfil longitudinal dos rios. Embora amplamente utilizado, sobretudo recentemente no Brasil, por vezes o índice não é plenamente entendido em seu sentido geomorfológico e em suas transformações matemáticas. O objetivo da presente nota é esclarecer tanto esse sentido quanto essas transformações. A abordagem adotada procura refazer o caminho lógico implícito na determinação do índice, propor procedimentos e analisar, criticamente, algumas recomendações de uso presentes na literatura. Os cálculos, propostas e análises são exemplificados com dados primários obtidos em um canal de baixa ordem e instalado sobre rochas vulcânicas.

Palavras-chave: Índice de gradiente; Perfil longitudinal; Geomorfologia fluvial.

Stream gradient index: meaning and application guidelines

\section{Abstract}

The gradient index is commonly used for evaluation of geologic control on the longitudinal profile of rivers. Although widely used, especially in Brazil recently, sometimes the index is not fully

Departamento de Geografia, Universidade Estadual do Centro-Oeste, UNICENTRO, Guarapuava, PR (adalto@unicentro.br).

Geosul, Florianópolis, v. 28, n. 56, p 147-162, jul./dez. 2013 
LIMA, A.G. Índice de gradiente de canal: significados e diretrizes para ... understood in its geomorphological meaning and in its mathematical transformations. The purpose of this note is to clarify both the meaning and the transformations. The approach seeks to remake the logical path implicit in the determination of the index, suggest procedures and analyze critically some usage recommendations found in the literature. The calculations, analyzes and proposals are exemplified with primary data obtained in a low order channel installed on volcanic rocks.

Key words: Gradient index; Longitudinal profile; Fluvial geomorphology.

\section{Introdução}

O índice de gradiente de canal (stream gradient index) foi proposto por Hack (1973) e, desde então, vem sendo designado por diversos nomes como índice de Hack e índice $S L$ ( $S$ de slope, ou declive, e $L$ de length ou extensão). No Brasil esse índice foi denominado de índice de declividade do canal (CHRISTOFOLETTI, 1981) e, mais recentemente, de relação declividade-extensão, ou RDE (ETCHEBEHERE et al., 2004). Na presente nota usa-se a expressão índice de gradiente de canal, ou apenas índice de gradiente, no sentido de ser mais fiel à denominação original e para evitar ambiguidade com outro índice utilizado no modelo que relaciona declive do canal e área de drenagem e que é denominado de índice de declividade (steepness index; WHIPPLE, 2000).

$\mathrm{O}$ índice de gradiente, embora amplamente usado, muitas vezes não é entendido em seu significado geomorfológico e suas possíveis transformações matemáticas podem ser fonte de equívocos conceituais e interpretativos. Nos escritos originais esses detalhes de significados e transformações não são tratados de forma suficiente. $\mathrm{O}$ objetivo dessa nota é esclarecer alguns pontos sobre o índice de gradiente, com vistas a contribuir para seu uso de modo adequado. Isso se faz oportuno, sobretudo ao considerar que 
LIMA, A.G. Índice de gradiente de canal: significados e diretrizes para ...

recentemente tem ressurgido no Brasil o interesse em se utilizar esse parâmetro geomorfológico para interpretação geológica (p. ex. ETCHEBEHERE et al., 2004; 2006; GUEDES et al., 2009; ROSANTE et al., 2010).

A abordagem adotada para a presente nota procura refazer o caminho lógico implícito na determinação do índice de gradiente. Primeiramente, é analisada a transformação logarítmica do perfil longitudinal dos canais e seu significado físico-morfológico. A partir da expressão matemática desse perfil, que constitui a base para o cálculo do índice de gradiente, é destacada sua relação com os parâmetros morfométricos do perfil convencional. São discutidos também os problemas de interpretação dessa equivalência. $\mathrm{Na}$ segunda parte é analisado o fator que constitui a essência da funcionalidade do índice, ou seja, a normalização das declividades. Finalmente, são analisadas algumas recomendações quanto ao uso do índice, encontradas em trabalhos que, embora basilares, não estendem a discussão e, portanto, geram dúvidas. Para esclarecer melhor as discussões, são utilizados como exemplo dados levantados pelo autor em um canal, aqui denominado de G2, de baixa ordem e esculpido em rochas vulcânicas da Formação Serra Geral, no Estado do Paraná.

\section{Perfis longitudinais e o índice de gradiente}

O índice de gradiente surgiu como uma alternativa de analisar os perfis longitudinais dos rios. Um perfil longitudinal de um canal de drenagem, entendido de modo convencional, é a relação entre a altimetria e a distância da nascente ou, em alguns casos, da foz (Figura 1 A). Hack (1973) propôs que esse perfil convencional fosse plotado de forma que o eixo $x$ (distância da nascente) estivesse em escala logarítmica e o eixo $y$ (altitudes), em escala aritmética (Figura $1 \mathrm{~B}$ ). 
LIMA, A.G. Índice de gradiente de canal: significados e diretrizes para ...

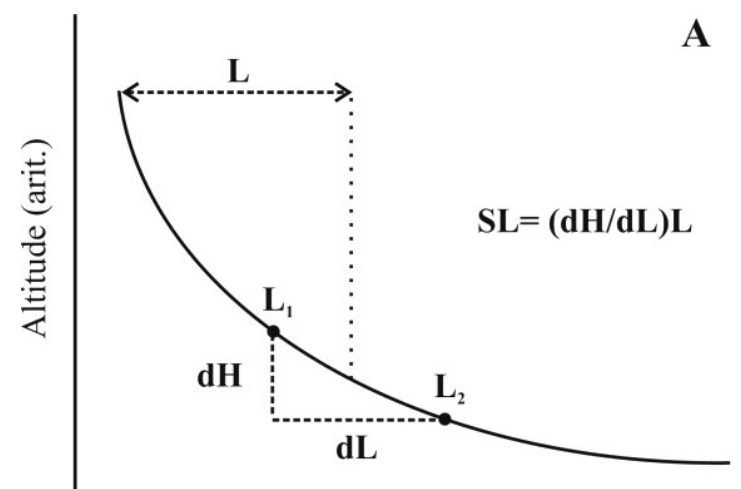

Distância da nascente (arit.)

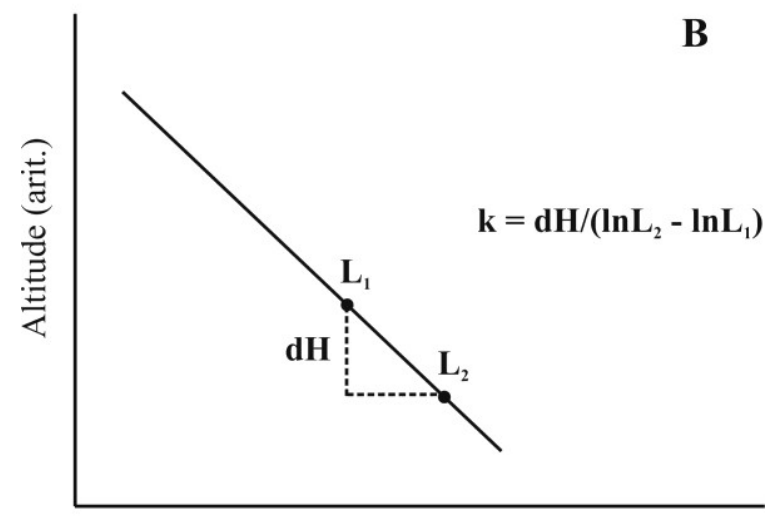

Distância da nascente $(\log )$

Figura 1: Perfis longitudinais esquemáticos. Em A, perfil convencional, em escalas aritméticas, e em B, perfil semilogarítmico. Ver texto para explicação dos símbolos. 
LIMA, A.G. Índice de gradiente de canal: significados e diretrizes para ...

A razão para esse procedimento de transformação está no fato de os perfis longitudinais tenderem para uma forma côncava, como resultado natural da evolução erosiva. O comprimento de cada trecho fluvial, situado entre dois níveis altimétricos distintos, tende a aumentar exponencialmente na medida em que se consideram distâncias maiores em relação ao ponto de origem do canal. Com a transformação proposta, o eixo $x$ em escala logarítmica mostra uma diminuição exponencial no comprimento dos trechos. O resultado gráfico ideal seria a formação de uma linha reta, composta de segmentos também retos (Figura $1 \mathrm{~B}$ ).

A transformação morfológica do perfil é um efeito da plotagem, que deriva da transformação matemática do eixo $x$, e isso implica em transformação de todos os parâmetros morfométricos que são calculados. Por exemplo, a partir dos dados do perfil convencional calcula-se a declividade de cada trecho do canal, considerando que:

$$
S=d H / d L
$$

onde, $S$ é a declividade, $d H$ é a diferença altimétrica entre dois pontos do perfil, e $d L$ é o comprimento do trecho. Porém, a partir dos dados do perfil semilogarítmico, se for aplicado o mesmo procedimento de cálculo, a declividade seria:

$$
S=d H / \ln L_{2}-\ln L_{1}
$$

onde, $\ln L_{2}$ e $\ln L_{1}$ são, respectivamente, os logaritmos da distância da nascente ao ponto final e inicial do trecho considerado. A declividade a partir da equação 2 não é a declividade real do canal, e sim a declividade do perfil semilogarítmico.

O índice de gradiente, tal como proposto por Hack (1973), deriva do perfil semilogarítmico e, de fato, corresponde à equação 2 , com a declividade $(S)$ sendo mais adequadamente descrita como $k$, ou seja, como índice de gradiente (equação 3; Figura 1).

$$
k=d H / \ln L_{2}-\ln L_{1}
$$


LIMA, A.G. Índice de gradiente de canal: significados e diretrizes para ...

Tabela 1: Dados morfométricos dos trechos do canal G2.

\begin{tabular}{rrrrrrr}
\hline $\boldsymbol{H}(\boldsymbol{m})$ & $\boldsymbol{D}(\boldsymbol{m})$ & $\boldsymbol{L}(\boldsymbol{m})$ & $\boldsymbol{S}$ & \multicolumn{1}{c}{$\boldsymbol{L}$} & $\boldsymbol{l n}$ & $\boldsymbol{k}$ \\
\hline 1235 & 0 & & & & & \\
1230 & 66 & 33,0 & 0,0758 & 2,50 & 4,1897 & \\
1225 & 123 & 94,5 & 0,0877 & 8,29 & 4,8122 & 8,03 \\
1220 & 145 & 134,0 & 0,2273 & 30,45 & 4,9767 & 30,39 \\
1215 & 156 & 150,5 & 0,4545 & 68,41 & 5,0499 & 68,38 \\
1210 & 178 & 167,0 & 0,2273 & 37,95 & 5,1818 & 37,90 \\
1205 & 193 & 185,5 & 0,3333 & 61,83 & 5,2627 & 61,80 \\
1200 & 208 & 200,5 & 0,3333 & 66,83 & 5,3375 & 66,80 \\
1195 & 221 & 214,5 & 0,3846 & 82,50 & 5,3982 & 82,47 \\
1190 & 237 & 229,0 & 0,3125 & 71,56 & 5,4681 & 71,53 \\
1185 & 251 & 244,0 & 0,3571 & 87,14 & 5,5255 & 87,12 \\
1180 & 271 & 261,0 & 0,2500 & 65,25 & 5,6021 & 65,22 \\
1175 & 295 & 283,0 & 0,2083 & 58,96 & 5,6870 & 58,92 \\
1170 & 322 & 308,5 & 0,1852 & 57,13 & 5,7746 & 57,09 \\
1165 & 344 & 333,0 & 0,2273 & 75,68 & 5,8406 & 75,65 \\
1160 & 393 & 368,5 & 0,1020 & 37,60 & 5,9738 & 37,55 \\
1155 & 435 & 414,0 & 0,1190 & 49,29 & 6,0753 & 49,24 \\
1150 & 478 & 456,5 & 0,1163 & 53,08 & 6,1696 & 53,04 \\
1145 & 565 & 521,5 & 0,0575 & 29,97 & 6,3368 & 29,90 \\
1140 & 653 & 609,0 & 0,0568 & 34,60 & 6,4816 & 34,54 \\
1135 & 765 & 709,0 & 0,0446 & 31,65 & 6,6399 & 31,59 \\
1130 & 820 & 792,5 & 0,0909 & 72,05 & 6,7093 & 72,02 \\
1125 & 927 & 873,5 & 0,0467 & 40,82 & 6,8320 & 40,77 \\
1120 & 1129 & 1028,0 & 0,0248 & 25,45 & 7,0291 & 25,36 \\
1115 & 1215 & 1172,0 & 0,0581 & 68,14 & 7,1025 & 68,11 \\
1110 & 1289 & 1252,0 & 0,0676 & 84,59 & 7,1616 & 84,57 \\
1105 & 1406 & 1347,5 & 0,0427 & 57,59 & 7,2485 & 57,55 \\
1100 & 1806 & 1606,0 & 0,0125 & 20,08 & 7,4989 & 19,97 \\
\hline & & & & & & \\
\hline
\end{tabular}

$H$ : altitude; $D$ : distância da nascente, neste caso, correspondendo à primeira curva de nível cruzada pelo canal; $L$ : extensão do canal, da nascente ao centro de um trecho; $S$ : declividade; $S L$ : produto da declividade pelo comprimento; ln: logaritmo natural; $k$ : índice de gradiente (cf. equação 3 ). 
LIMA, A.G. Índice de gradiente de canal: significados e diretrizes para ...

Para o canal integral $L_{l}$ pode ser igual a 1 metro, o que resultaria $\ln L_{1}=0$ e $L_{2}$ seria o comprimento total do canal. Portanto, $\ln L_{2}-\ln L_{1}$ (equação 3) seria igual ao logaritmo de $d L$, ou simplesmente $\ln L_{2}$. Essa igualdade, porém, é válida somente para o canal como um todo. Para os trechos, $L_{l}$ sempre será maior que 1 metro e $\ln L_{1}$ será diferente de zero, de modo que os erros ao se usar apenas $\ln 2$ na equação 3 , podem ser significativos, como se deduz a partir dos dados da Tabela 1: a diferença entre dois $\ln$ consecutivos $\left(\ln _{2}-\ln _{1}\right)$ é sempre menor que $\ln _{2}$.

O segmento inicial de muitos canais apresenta influência coluvial marcante. Somente após certa extensão os processos fluviais passam a predominar. A distinção entre os segmentos é identificada pela mudança na taxa de diminuição da declividade rio abaixo, muito menor no segmento inicial (SKLAR e DIETRICH, 1998; STOCK e DIETRICH, 2003; DUVALL et al., 2004). A origem do canal deve ser considerada como o ponto onde os processos fluviais começam a predominar. No entanto, não há como determinar o ponto de transição sem plotar a declividade contra a distância da nascente. Dependendo da escala de trabalho, esse corte do canal pode estar incluso na simplificação cartográfica. Este é o caso das escalas menos detalhadas que 1: 25.000, nas quais a origem do canal pode coincidir com o início da linha azul. Em escalas de detalhe e semidetalhe, se não for determinado o ponto de transição do canal, o cálculo do índice de gradiente (equação 3) para o canal integral, pode incluir um erro cuja magnitude dependerá do comprimento do segmento inicial e da sua respectiva amplitude altimétrica. Este aspecto é particularmente importante quando se pretende comparar o índice geral do canal com os índices dos trechos.

A Figura 2 mostra os perfis do canal G2 e a relação declividade-distância da nascente, conforme dados da Tabela 1. Os primeiros 120 metros não se ajustam à tendência das declividades do restante do canal. Neste caso, diferente dos reportados na literatura, o segmento inicial possui declividades relativamente 
LIMA, A.G. Índice de gradiente de canal: significados e diretrizes para ... baixas. Isso está relacionado à interação coluvio-aluvial e também à litologia, pois o canal transiciona de riodacitos para basaltos.
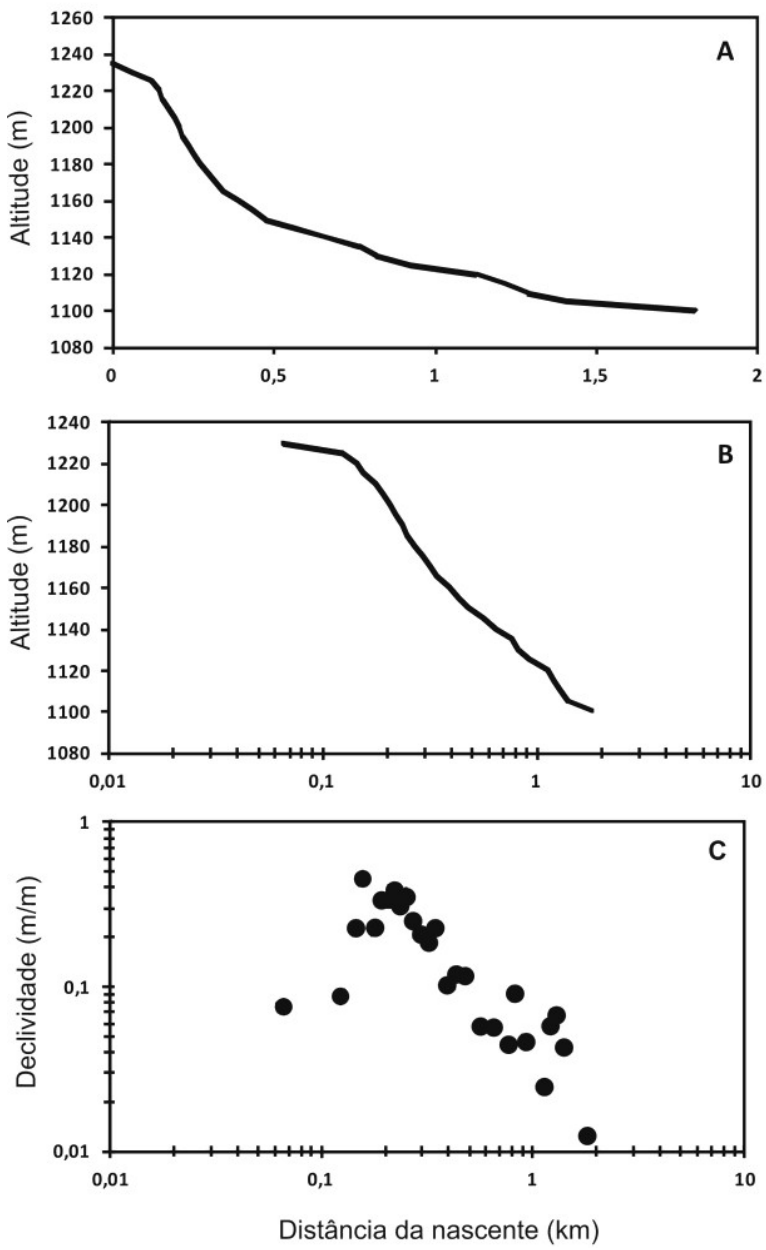

Figura 2: Perfis longitudinais e relação declividade-distância do canal G2. 
LIMA, A.G. Índice de gradiente de canal: significados e diretrizes para ...

$\mathrm{Na}$ Tabela 2 estão os resultados do cálculo do índice de gradiente para o canal G2 integral. Em $k 1$ o segmento inicial é descartado por dois modos: (a) considerando o canal apenas depois da interseção com a primeira curva de nível e (b) considerando o canal apenas depois da mudança morfológica identificada na Figura $2 \mathrm{C}$. Em ambos os casos tem-se $L_{1} \neq 1 \mathrm{~m}$, porque a contagem do comprimento do canal começa na nascente. Em $k 2$ o segmento inicial também é descartado pelos dois modos, porém tem-se $L_{l}=1 \mathrm{~m}$. Primeiramente, $\mathrm{o}$ índice $k$ pode variar apreciavelmente conforme se adote $L_{1} \neq 1$ ou $L_{1}=1$. Se for adotado $L_{l}=1$, o método para identificar e descartar o segmento inicial não tem efeito apreciável sobre o valor do índice. Desse modo é recomendável que se adote $L_{1}=1$ para minimizar diferenças oriundas de eventual consideração errônea do ponto de transição morfológica do perfil.

Note-se, portanto, que ao comparar canais distintos a base de cálculo deve ser a mesma, para evitar que as diferenças sejam atribuídas a algum fator físico, como a geologia. Comparar o índice geral com os índices dos trechos de um canal também exige a mesma base de cálculo. Na Tabela 1 os cálculos de $l n$ e $k$ dos trechos, estão atrelados à coluna referente à distância da nascente $(D)$. Isso significa que se $L_{l}=1$ for adotado para o índice do canal integral, a coluna $D$ precisa ser recalculada a partir do ponto onde se considere a transição morfológica do perfil, fazendo-se esse ponto igual a 1 .

Tabela 2: Dados morfométricos do canal G2 integral.

\begin{tabular}{ccccccc}
\hline $\boldsymbol{d} \boldsymbol{H}$ & $\boldsymbol{d} \boldsymbol{L}$ & $\boldsymbol{S}$ & $\boldsymbol{L}$ & $\boldsymbol{S} \boldsymbol{L}$ & $\boldsymbol{k} \boldsymbol{1}$ & $\boldsymbol{k 2}$ \\
\hline 130 & 1740 & 0,0747 & 870,0 & 65 & 39,28 & 17,42 \\
120 & 1661 & 0,0722 & 830,5 & 60 & 47,58 & 16,18 \\
\hline
\end{tabular}

$d H$ : diferença altimétrica $(\mathrm{m}) ; d L$ : extensão total do canal; $S$ : declividade; $S L$ : índice de gradiente; $k 1$ :, índices de gradiente calculados considerando-se, respectivamente, $L_{l}=33$ e 134, ou seja, a origem do canal é a fonte, igual a zero; $k 2$ : índices de gradiente calculados considerando-se $L_{I}=1$. 
LIMA, A.G. Índice de gradiente de canal: significados e diretrizes para ...

Para traduzir a equação 3 em termos do perfil convencional considere-se que:

$$
S L \approx d H / \ln L_{2}-\ln L_{1}
$$

Em outras palavras, o índice de gradiente é aproximadamente igual ao produto da declividade pela extensão da nascente ao centro do trecho considerado (Figura 1). A Tabela 1 mostra exemplos dessa diferença, que não é percebida senão no nível decimal. Entretanto, o destaque é necessário ao se considerar que no texto original de Hack (1973) a igualdade é assumida como absoluta e que, por conseguinte, outra expressão (equação 5) pode ser fonte de equívocos.

Para expressar a composição de $S L$ é possível distinguir os seus elementos componentes:

$$
S L=(d H / d L) L
$$

Se houvesse igualdade absoluta na equação 4, o segundo membro da equação 5 seria igual ao segundo membro da equação 4. Note-se, entretanto, que o segundo membro da equação 4 é definido no perfil semilogarítmico, enquanto o da equação 5 , no perfil convencional. Ou seja, um é o declive do perfil semilogarítmico e o outro é o produto da declividade real pela distância da nascente.

Devido à aproximada igualdade expressa na equação 4 , o índice de gradiente $(k)$ pode ser também chamado de índice $S L$, ou vice versa. $\mathrm{Na}$ literatura geomorfológica brasileira, tem sido comum o uso da denominação abreviada de $R D E$, ou seja, Relação Declividade-Extensão (ETCHEBEHERE et al., 2004; 2006). Entendido pelo significado estrito, o termo relação diria respeito ao comportamento combinado entre as variáveis declividade $(S)$ e extensão $(L)$, quando plotadas uma contra a outra. Porém, não é esse o caso na definição do índice de gradiente. Aparentemente houve a intenção de usar o termo relação para significar indiretamente o produto entre as variáveis.

Para evitar tais confusões, na presente nota prefere-se não dar continuidade ao uso da expressão $R D E$. Por outro lado, apenas 
LIMA, A.G. Índice de gradiente de canal: significados e diretrizes para ...

para efeito das discussões aqui articuladas é conveniente manter a distinção da nomenclatura entre índice de gradiente $(k)$ e índice $S L$.

\section{Normalização da declividade}

A grande conquista da proposta de Hack (1973) está no fato da declividade de um trecho ser normalizada pela distância da nascente, seja através do produto $S L$ seja através da transformação logarítmica. Somente desse modo a declividade poderá ser comparada com a de outros trechos, mesmo que estes estejam distantes entre si. Para apreciar melhor o significado da transformação, considere-se que a declividade tende a diminuir rio abaixo em um perfil côncavo devido a um ajuste hidráulico e sedimentológico (SINHA e PARKER, 1996; KNIGHTON, 1998; SKLAR e DIETRICH, 2008). A normalização pela distância elimina as diferenças oriundas da posição no perfil - ou do controle hidráulico e sedimentológico - deixando realçar as diferenças devidas ao controle geológico.

A distância da nascente, pela qual é feita a normalização da declividade de um trecho, é considerada como a distância da origem do canal até o ponto médio entre os dois pontos delimitantes do trecho (Hack, 1973; Figura 1 A). Em alguns trabalhos, como Etchebehere et al., (2004; 2006), essa distância tem sido tomada até o ponto final do trecho considerado, ou seja, em $L_{2}$. Como resultado, a tendência é de os valores serem maiores, porque a distância (ou extensão, $L$ ) é ampliada.

Quando se está trabalhando apenas com a análise de $S L$ dos trechos, a mudança de parâmetro não tem importância. No entanto, se a análise envolver uma comparação dos trechos com o índice de gradiente $(k)$ do canal integral, que fora obtido pelos logaritmos (ver discussão na próxima seção), talvez a diferença possa ser um fator a ser considerado com mais cuidado. A diferença entre o índice do canal integral e os índices $S L$ dos trechos pode ser maior ou menor, dependendo do critério adotado. 
LIMA, A.G. Índice de gradiente de canal: significados e diretrizes para ...

\section{Combinação SL e k}

No trabalho de Hack (1973) a igualdade entre $S L$ e $k$ é constatada, no entanto apenas $S L$ é usado como fonte de cálculo. Seeber e Gornitz (1982), ao estudarem os perfis longitudinais de rios da cordilheira do Himalaia, inovaram ao combinarem $S L$ e $k$. Para cada canal esses pesquisadores calcularam o índice de gradiente $(k)$, considerando a sua extensão total, e o índice $S L$ para os diversos trechos em que o canal foi dividido. Para detecção de possíveis anomalias tectônicas o índice $S L$ de cada trecho foi dividido pelo índice de gradiente. Algumas questões podem surgir frente ao emprego desse procedimento e que o texto de Seeber e Gornitz (1982) não esclarece: Poderia ser usado o índice $S L$ para o canal como um todo, ou então o índice $k$ para cada trecho?

Para melhor compreensão dessas questões é necessário observar os dados da tabela 1. O índice $S L$ difere significativamente do índice de gradiente, quando aplicados ao canal integral. O cálculo de $S L$ é baseado na declividade geral do canal, o que não é um parâmetro morfológico realista. Considerese que o perfil, no exemplo da Tabela 1, possui forma aproximadamente côncava (Figura 2). A declividade, portanto, diminui sistematicamente rio abaixo e uma declividade geral é apenas uma abstração grosseira. Por outro lado, o cálculo de $k$ resulta na declividade do perfil semilogarítmico, admitida a concavidade como forma ideal. No caso do canal G2, o real aproxima-se do ideal. Mesmo que não houvesse essa aproximação, o índice $k$ representaria o declive ideal, contra o qual os valores reais seriam comparados. Em outras palavras, o declive calculado apenas com os dados da diferença altimétrica $(d H)$ e do logaritmo do comprimento total do canal $\left(\ln L_{2}-\ln L_{1}\right)$ é a tradução de um perfil ideal côncavo. A declividade geral de um perfil convencional, por sua vez, é irreal e geomorfologicamente quase impossível. Portanto, o uso do índice $S L$ para o canal integral, como fizeram Guedes et al. (2009) e Rosante et. al. (2010), é 
LIMA, A.G. Índice de gradiente de canal: significados e diretrizes para ...

matematicamente possível, porém constitui um erro, por não possuir uma base conceitual aceitável.

A utilização de $S L$ para trechos relativamente menores de um canal é mais factível, embora também careça de uma base real, pois não há trechos, senão muito pequenos, com declive constante.

Sua facilidade de cálculo e a quase igualdade com o índice de gradiente o torna a alternativa mais prática para uso em trechos de um canal. Note-se que quanto maior é a diferença altimétrica entre os pontos inicial e final de um trecho, maior será a diferença dos valores de $S L$ e $k$, porque menos real se torna a declividade medida.

O índice de gradiente $(k)$ é mais consistente em termos conceituais do que o índice $S L$ e em qualquer situação - trecho ou canal integral - constitui-se na alternativa mais correta. Em resumo, a recomendação seria ou usar apenas a formulação de $k$ ou, como fizeram Seeber e Gornitz (1982), aplicar $S L$ nos trechos e $k$ no perfil como um todo.

\section{Conclusões}

O índice de gradiente é uma alternativa para análise mais eficiente do controle exercido por fatores geológicos sobre o perfil longitudinal de um canal de drenagem. Ele deriva do perfil semilogarítmico, o que evita o efeito da diminuição da declividade pelos fatores hidráulicos e sedimentológicos.

$\mathrm{O}$ índice corresponde à declividade do perfil semilogarítmico e equivale ao produto da declividade do perfil convencional pela distância da nascente. No entanto, essa equivalência é apenas aproximada e válida somente para trechos do perfil longitudinal. Para o perfil integral a declividade real pode se distanciar muito da declividade média utilizada no cálculo de $S L$ e o resultado será muito diferente do obtido no cálculo pelos logaritmos $(k)$. Recomenda-se, então, usar apenas a formulação de $k$, tanto em trechos como no perfil integral, ou aplicar $S L$ nos 
LIMA, A.G. Índice de gradiente de canal: significados e diretrizes para ...

trechos e $k$ no perfil integral, como fizeram Seeber e Gornitz (1982).

O cálculo do índice do canal integral pelos logaritmos pode ser feito considerando-se $L_{l} \neq 1$ ou $L_{l}=1$ e sem o segmento inicial do canal, que geralmente possui comportamento morfológico distinto devido aos processos nele operante. Adotando-se $L_{1}=1$, o método para identificar e descartar o segmento inicial não tem efeito apreciável sobre o valor do índice.

Ao utilizar dados do perfil convencional (SL), a normalização da declividade deve ser feita como recomendada por Hack (1973), ou seja, pela distância entre a nascente e o ponto médio do trecho considerado.

\section{Referências bibliográficas}

CHRISTOFOLETTI, A. Geomorfologia fluvial. São Paulo: Blücher, 1981. 313 p.

DUVALL, A.R.; KIRBY, E.; BURBANK, D.W. Tectonic and lithologic controls on bedrock channel profiles and processes in coastal California. J. Geoph. Research, v.109, F03002, 18 p., doi:10.1029/2003JF000086, 2004.

ETCHEBEHERE, M. L.; SAAD, A.R.; FÚlFARO, V.J. PERINOTTO, J.A.J. Aplicação do índice "Relação DeclividadeExtensão - RDE" na Bacia do Rio do Peixe (SP) para detecção de deformações neotectônicas. Geol. USP Sér. Cient., São Paulo, v. 4, n. 2, p. 43-56, 2004.

ETCHEBEHERE, M. L.; SAAD, A.R.; SANTONI, G.; CASADO, F.C.; FULFARO, V. J. Detecção de prováveis deformações neotectonicas no vale do rio do Peixe, região Ocidental Paulista, mediante aplicação de índices RDE (Relação Declividadeextensão) em segmentos de drenagem. Geociências, São Paulo, v. 25, n. 3, p. 271-287, 2006. 
LIMA, A.G. Índice de gradiente de canal: significados e diretrizes para ...

GUEDES, I.C.; ETCHEBEHERE, M.L.C.; MORALES, N.; STEVAUX, J.C.; SANTONI, G.C. Análise morfotectônica da bacia do rio Santo Anastácio, SP, através de parâmetros fluviomorfométricos e de registros paleossísmicos. Geociências, São Paulo, v. 28, n. 4, p. 247-362, 2009.

HACK, J.T. Stream profile analysis and stream gradient index. J. Res. US Geol. Survey, v.1, n.4, p.421-429, 1973.

KNIGHTON, A.D. Fluvial forms and processes: a new perspective. Arnold: Londres, 1998. 383p.

ROSANTE, K.T.; HACKSPACHER, P.C.; DORANTI-TIRITAN, C.; GODOY, D.F. Análise morfométrica da bacia do rio Capivari (alto Rio Pardo) como contribuição aos estudos de neotectônica do planalto sul de Minas. Revista de Geografia. Recife, v. especial VIII SINAGEO, n. 1, p. 128-139, 2010.

SEEBER, L.; GORNITZ, V. River profiles along the Himalayan arc as indicators of active tectonics. Tectonophysics, v. 92, p. 335$367,1982$.

SINHA, S.K.; PARKER, G. Causes of concavity in longitudinal profiles of rivers. Water Res. Research, v. 32, n. 5, p. 1417-1428, 1996.

SKLAR, L.; DIETRICH, W.E. River longitudinal profiles and bedrock incision models: stream power and the influence of sediment supply. In: TINKLER, K. J. e WOHL, E.E (Org.). Rivers over rock: fluvial processes in bedrock channels. American Geophysical Union: Washington, DC, 1998, p. 237-260.

SKLAR, L.; DIETRICH, W.E. Implications of the saltationabrasion bedrock incision model for steady-state river longitudinal 
LIMA, A.G. Índice de gradiente de canal: significados e diretrizes para ... profile relief and concavity. Earth Surf. Proc. Landforms, v. 33, n. 7, p. $1129-1151,2008$

STOCK, J.; DIETRICH, W. E. Valley incision by debris flows: Evidence of a topographic signature, Water Resour. Res., v. 39, n.4, 1089, doi:10.1029/2001WR001057. 2003.

WHIPPLE, K.X. Bedrock rivers and the geomorphology of active orogens. Annu. Rev. Earth Planet. Sci., v. 32, p. 151-185, 2004.

Recebido em fevereiro de 2013

Aceito em outubro de 2013 